\title{
BRS Cohomology and Topological Anomalies
}

\author{
Gregorio Falqui and Cesare Reina * \\ Dipartimento di Fisica dell'Università, Via Celoria 16, I-20133 Milano, Italy
}

\begin{abstract}
The occurrence of non-abelian anomalies in gauge theories and gravitation, first discovered via perturbative techniques, is now completely explained from the mathematical point of view by means of the family index theorem of Atiyah and Singer. Here we make contact between this approach and BRS cohomology, by showing that they yield the same non-abelian anomalies, provided a certain restriction to "local" functionals is not introduced from the very beginning. In particular, this solves the "unicity" problem for this kind of anomalies. Local BRS cohomology is still relevant for the abelian case.
\end{abstract}

\section{Introduction}

As it is well known, the conservation of certain fermionic currents in gauge theories does not survive quantization, giving rise to the so-called anomalies. These were first discovered via Feynman diagram techniques [1], but have been successively studied by a variety of tools.

First, a cohomological interpretation was found [2] thanks to the nilpotency of the BRS operator $\delta$. A functional $a=a(A, \omega)$ (locally depending on the gauge potential $A$ and linear in the ghost $\omega$ ) is called an anomaly if it satisfies the WessZumino consistency condition $\delta a=0$, but there is no local functional $\Lambda_{\mathrm{loc}}(A)$ such that $-\delta \Lambda_{\mathrm{loc}}(A)=a$. In physical terms this implies that there is no redefinition $\Gamma \rightarrow \Gamma+\Lambda_{\text {loc }}$ of the "effective action" $\Gamma$ which cancels the anomaly itself. Clearly, the problem is of cohomological nature, and a proper mathematical set up involves the cohomology of the Lie algebra of infinitesimal gauge transformations with local functionals of the gauge potentials as coefficients [3]. We call this cohomology the "local" BRS-cohomology.

Soon after the cohomological nature of anomalies was understood, their relations with the secondary characteristic classes of Chern and Simons was

* Work partially supported by Gruppo Nazionale di Fisica Matematica del CNR and Progetto Nazionale "Geometria e Fisica" del MPI 
discovered [4]. This was mainly an "algebraic" development, showing that the "Russian formula" yields exactly the same anomalies as found by physicists via perturbative techniques. Still one does not know if such a construction exhausts the relevant BRS-cohomology group, that is if there are more solutions of the equations $\delta a=0, a \neq \delta \Lambda_{\text {loc }}$ than the anomalies found perturbatively. In other words, we have a "unicity" problem.

As characteristic classes are intimately related with the topology of fibre bundles [5], it was natural to inquire as to what topological structure was actually controlling a piece of quantum field theory. For abelian anomalies the answer was quite readily given [6] in terms of the Atiyah-Singer index theorem: it was the chiral asymmetry of the zero modes of the Dirac operator that breaks conservation of the abelian chiral current. Non-abelian anomalies had to wait longer, until recently a subtle application of the index theorem for families of operators showed $[7,8]$ their topological meaning. This time it is the twisting of the infinite dimensional bundle of gauge orbits (that is the presence of Gribov ambiguity) that produces non-abelian anomalies. More precisely these are due to the non-triviality of the determinant line bundle of the index bundle for the family of Dirac operators parametrized by gauge potentials [7].

The relation between this topological set up for non-abelian anomalies and the physicist's way of constructing them have been studied in some papers [8-10,23], and it is by now fairly well understood. One sees that when the index bundle has a non-trivial first Chern class, treating zero modes in the computation of the determinant of the Dirac operator becomes a nasty global question. In particular, the phase of the determinant is not single valued, preventing the existence of a well defined effective action $\Gamma=\Gamma(A)$, either local or not, such that $a=\delta \Gamma$. That is why the occurrence of this kind of anomalies is so harmful in quantum field theory. The family index theorem also tells us that these obstructions to a well defined quantum theory have little to do with perturbation theory, their basic spring being topology. So one can safely forget the rules of these techniques, among which "locality" of functionals.

In this paper we study the relations between the cohomological description and the topological nature of non-abelian anomalies. This is technically done by considering a suitable group cohomology, whose "infinitesimal" version coincides with (non-local) BRS cohomology. The main point is that we do not restrict ourselves to local functionals, but we work with the whole space of complex valued smooth functionals as coefficients, complexification being essential for explaining non-abelian anomalies. This enlargement of coefficients "dilutes" cohomology in such a way that the relevant BRS cohomology group turns out to be isomorphic with the first Chech cohomology group $H^{1}(\mathscr{G}, Z)$ of the group of gauge transformations. This is in full agreement with the index theorem approach.

\section{Cohomological Description of Anomalies}

The main object of our study is the regularized vacuum functional $W(A)$ of some matter field in an external SU $(n)$ gauge field $A$, on the compactified euclidean space time $S^{4}$. The space $\mathscr{A}$ of these external gauge fields is an affine space; so it has no 
homotopy nor homology. Hereafter we feel it is easier to work in some suitable Sobolev functional space [11], such that $\mathscr{A}$ is a Hilbert manifold. Then the vacuum functional is a map

$$
W: \mathscr{A} \rightarrow \mathbb{C},
$$

which in physical applications can be assumed to be smooth, the main physical example being the vacuum functional for chiral fermions $[9,10]$.

In physics, one is interested in the effective action $\Gamma(A)=\log W(A)$, although, as we shall see, taking this logarithm may be an ill-defined operation. In any case, assuming that the effective action exists as a smooth functional on $\mathscr{A}$, one needs to study its transformation properties under gauge transformations. Recall that the Hilbert-Lie group $\mathscr{G}$ of (pointed) gauge transformations in some suitable Sobolev class acts freely and smoothly on $\mathscr{A}$ on the right by $A \rightarrow A g$, where $A g$ is the gauge potential obtained from $A$ under the gauge transformation $g \in \mathscr{G}$. Recall also that the space $\mathcal{O}=\mathscr{A} / \mathscr{G}$ of classes of gauge-equivalent potentials can be given the structure of a smooth Hilbert manifold. Then the map $\pi: \mathscr{A} \rightarrow \mathcal{O}$ turns out to be a principal fibre bundle with structure group $\mathscr{G}$. We refer for instance to [12] for a review of this geometrical set up.

As $\mathscr{G}$ acts smoothly on $\mathscr{A}$, one can study the infinitesimal variation of $\Gamma(A)$ by considering a one parameter subgroup $g_{t}=\exp (t \tau)$, generated by an element $\tau$ of the Lie algebra $\mathfrak{g}$ of $\mathscr{G}$, and setting

$$
\delta_{\tau} \Gamma(A)=\lim _{t \rightarrow 0}\left(\Gamma\left(A g_{t}\right)-\Gamma(A)\right) / t=: a(\tau ; A) .
$$

Now, $a(\tau ; A)$ can be considered as a linear map of $\mathrm{g}$ into the space $\mathbb{C}(\mathscr{A})$ of complex valued smooth functionals on $\mathscr{A}$. It is called an (integrated) anomaly whenever there is no local redefinition $\Gamma^{\prime}=\Gamma+\Lambda_{\mathrm{loc}}$ of the effective action, such that $\delta_{\tau} \Gamma^{\prime}=0$. Clearly, this requires the solution of the equation $\delta_{\tau} \Lambda_{\mathrm{loc}}=-a$, which, as it is well known [2], may be impossible because of cohomological obstructions.

Instead of working infinitesimally, one can consider a finite version of Eq. (2), by directly studying the transformation properties of $W$. That is one sets [13];

$$
W(A g)=\exp (2 \pi i F(g, A)) W(A),
$$

where now $F: \mathscr{G} \rightarrow \mathbb{C}(\mathscr{A})$ is assumed to be a smooth map. Again, the definition of $F$ requires taking a logarithm, which may be not well defined. In physical applications $F(g, A)$ turns out to depend locally on $A$. Now $2 \pi i \lim _{t \rightarrow 0} F$ $\cdot\left(g_{t}, A\right) / t$ yields directly the same anomaly as Eq. (2). The functional $F(g, A)$ is usually called the Wess-Zumino term.

As already remarked, both these descriptions of the anomalies heavily rely on the possibility of defining logarithms. It may, however, happen that the non-trivial topology of $\mathscr{G}$ prevents the existence of the single valued logarithms one wants to compute. As we shall see, this is the basic topological nature of non-abelian anomalies. In order to avoid logarithms, we prefer to work with a "multiplicative" group cohomology, rather than with the "additive" one implicitly required by Eq. (2).

To be definite, we denote by $\mathbb{C}^{*}(\mathscr{A})$ the abelian group of never vanishing complex valued smooth functionals on $\mathscr{A}$, the group operation being pointwise 
multiplication $\left(N_{1} N_{2}\right)(A)=N_{1}(A) N_{2}(A)$. Since $\mathscr{G}$ acts on $\mathscr{A}$, it also naturally acts on $\mathbb{C}^{*}(\mathscr{A})$ by pull-back, i.e. we have $\left(g^{*} N\right)(A)=N(A g)$. A $k$-cochain on $\mathscr{G}$ with values in $\mathbb{C}^{*}(\mathscr{A})$ is a smooth map

$$
f_{k}: \mathscr{G} \times \ldots \times \mathscr{G} \rightarrow \mathbb{C}^{*}(\mathscr{A}),
$$

depending on $k$ arguments. A coboundary operator $\Delta$ is then defined by

$$
\begin{aligned}
\left(\Delta f_{k}\right)\left(g_{1}, \ldots, g_{k+1} ; A\right)= & f_{k}\left(g_{2}, \ldots, g_{k} ; A g_{1}\right) f_{k}\left(g_{1}, \ldots, g_{k} ; A\right)^{(-1)^{k+1}} \\
& \cdot \prod_{i=1}^{k} f_{k}\left(g_{1}, \ldots, g_{i-1}, g_{i} g_{i+1}, \ldots, g_{k} ; A\right)^{(-1)^{i}} .
\end{aligned}
$$

A $k$-cochain $f_{k}$ is called a $k$-cocycle if $\Delta f_{k}=1$. Notice that we have 1 instead of 0 , according to the multiplicative nature of $\mathbb{C}^{*}(\mathscr{A})$. It is a coboundary if $f_{k}=\Delta f_{k-1}$, for some $f_{k-1}$ nowhere vanishing. As $\Delta^{2}=1$, any coboundary is a cocycle, but the converse may not be true. As usual, one introduces the group $H^{k}\left(\mathscr{G}, \mathbb{C}^{*}(\mathscr{A})\right)$ of $k$-cocycles modulo $k$-coboundaries, which is called the $k^{\text {th }}$ cohomology group of $\mathscr{G}$ with coefficients in $\mathbb{C}^{*}(\mathscr{A})$. In particular, a 0 -cochain is simply a functional $N \in \mathbb{C}^{*}(\mathscr{A})$; its coboundary reads

$$
(\Delta N)(g ; A)=N(A g) / N(A) .
$$

So a 0 -cocycle is a $\mathscr{G}$-invariant functional in $\mathbb{C}^{*}(\mathscr{A})$. A 1 -cochain $f=f(g ; A)$ is a cocycle if

$$
(\Delta f)\left(g_{1}, g_{2} ; A\right)=f\left(g_{2} ; A g_{1}\right) f\left(g_{1} ; A\right) / f\left(g_{1} g_{2} ; A\right)=1 \text {. }
$$

Let us go back to the generating functional $W$. In physical problems $W$ vanishes along $\mathscr{G}$-orbits in $\mathscr{A}$, if any, i.e. $W(A g)=0$ if and only if $W(A)=0$. We assume that the functional

$$
f(g ; A)=: W(A g) / W(A)
$$

is well defined and never vanishing on $\mathscr{G} \times \mathscr{A}$. There is a number of evidencies that this is indeed the case in physics $[9,10]$. So $f$ defines a 1 -cochain on $\mathscr{G}$ with values in $\mathbb{C}^{*}(\mathscr{A})$. The following proposition is easily proved

Proposition 2.1. For any $g \in \mathscr{G}$, let $f(g ; A)=W(A g) / W(A)$ be a never vanishing functional on $\mathscr{A}$. Then

i) $f$ is a 1-cocycle of $\Delta$, and defines a cohomology class $[f] \in H^{1}\left(\mathscr{G}, \mathbb{C}^{*}(\mathscr{A})\right)$,

ii) $f$ is a coboundary if and only if $W$ is never vanishing on $\mathscr{A}$.

Proof. As for i), we compute from Eq. (8),

$$
\begin{array}{r}
f\left(g_{1} g_{2} ; A\right)=W\left(A g_{1} g_{2}\right) / W(A)=W\left(A g_{1} g_{2}\right) / W\left(A g_{1}\right) \\
\cdot W\left(A g_{1}\right) / W(A)=f\left(g_{2} ; A g_{1}\right) f\left(g_{1} ; A\right),
\end{array}
$$

then Eq. (7) tells us that $\Delta f=1$. As for ii), notice that if $W$ is never vanishing, it is a 0 -cochain on $\mathscr{G}$ with values in $\mathbb{C}^{*}(\mathscr{A})$ and $f=\Delta W$. Conversely, if $[f]=1$, then there exists a representative $f^{\prime} \in[f]$ (i.e. an $f^{\prime}=f \Delta N$ ) such that $f^{\prime}=\Delta W^{\prime}$ for some never vanishing $W^{\prime}=W^{\prime}(A)$. Then

$$
W^{\prime}(A g) / W^{\prime}(A)=f^{\prime}(g ; A)=f(g ; A)(\Delta N)(g ; A)=W(A g) N(A g) /(W(A) N(A))
$$


implies that $W$ and $W^{\prime}$ have the same zeroes and, since $W^{\prime}$ is never vanishing, so is $W$.

Remark. Notice that, if $f$ is a coboundary, the $\operatorname{logarithm} \Gamma=\log W$ is a smooth functional on $\mathscr{A}$. In fact, the space of gauge potentials is contractible and the logarithm of a never vanishing complex valued function on a simply connected space is well defined.

From Proposition 2.1 it is clear that there should be some relation between the cohomology class $[f]$ and the zeroes of $W$. We can be more precise on this point, by showing that any representative $f$ of $[f] \in H^{1}\left(\mathscr{G}, \mathbb{C}^{*}(\mathscr{A})\right)$ can be written as $f=W(A g) / W(A)$ for some suitable functional $W: \mathscr{A} \rightarrow \mathbb{C}$ (see Proposition 3.2). This requires the introduction of a line bundle over the orbit space $\mathcal{O}$, as will be described in Sect. 3.

Next we want to make contact with the Lie algebra cohomology mentioned above, without introducing the functional $\log W$. First of all recall [3] that a $k$-cochain on the Lie algebra $\mathfrak{g}$ of $\mathscr{G}$ with coefficients in the space $\mathbb{C}(\mathscr{A})$ of complex valued functionals on $\mathscr{A}$ is simply a $k$-linear skew map

$$
a_{k}: \mathfrak{g} \times \ldots \times \mathfrak{g} \rightarrow \mathbb{C}(\mathscr{A}) .
$$

For any $\tau \in \mathfrak{g}$, define the operator $\mathscr{L}_{\tau}$ by

$$
\mathscr{L}_{\tau} a_{k}\left(\tau_{1} \ldots \tau_{k} ; A\right)=: \lim _{t \rightarrow 0}\left[a_{k}\left(\tau_{1} \ldots \tau_{k} ; A g_{t}\right)-a_{k}\left(\tau_{1} \ldots \tau_{k} ; A\right)\right] / t,
$$

where $g_{t}=\exp (t \tau)$ as above. In other terms, $\mathscr{L}_{\tau}$ is the Lie derivative of $a_{k}$ considered as a functional on $\mathscr{A}$ with respect to the fundamental vector field on $\mathscr{A}$ generated by $\tau$. Then one constructs a coboundary operator $\delta$ as follows:

In particular, one has

$$
\begin{aligned}
\left(\delta a_{k}\right)\left(\tau_{1} \ldots \tau_{k+1} ; A\right)= & : \sum_{i=1}^{k+1}(-1)^{i+1} \mathscr{L}_{\tau_{i}} a_{k}\left(\tau_{1} \ldots \hat{\tau}_{i} \ldots \tau_{k+1}\right) \\
& +\sum_{i<j}(-1)^{i+j} a_{k}\left(\left[\tau_{i}, \tau_{j}\right] \tau_{1} \ldots \hat{\tau}_{i} \ldots \hat{\tau}_{j} \ldots \tau_{k+1}\right) .
\end{aligned}
$$

$$
\begin{aligned}
\left(\delta a_{0}\right)(\tau ; A) & =\mathscr{L}_{\tau} a_{0}(A), \\
\left(\delta a_{1}\right)\left(\tau_{1} \tau_{2} ; A\right) & =\mathscr{L}_{\tau_{1}} a_{1}\left(\tau_{2} ; A\right)-\mathscr{L}_{\tau_{2}} a_{1}\left(\tau_{1} ; A\right)-a_{1}\left(\left[\tau_{1}, \tau_{2}\right] ; A\right) .
\end{aligned}
$$

Now $\delta^{2}=0$ and, as usual, one defines the groups $H^{k}(\mathfrak{g}, \mathbb{C}(\mathscr{A}))$ by taking $k$-cocycles (i.e. $a_{k}$ 's such that $\delta a_{k}=0$ ) modulo $k$-coboundaries (i.e. $a_{k}$ 's such that $a_{k}=\delta a_{k-1}$, for some $\left.a_{k-1}\right)$. We call this cohomology of the Lie algebra $g$ of the infinitesimal gauge transformation the BRS-cohomology, since the operator $\delta$ can be identified with the BRS operator, when one replaces anticommutativity of ghosts with skew symmetrization of infinitesimal gauge transformations [14].

We now prove that working with the group or the algebra cohomologies introduced above is quite the same, at least at the level of the first cohomology groups which describe anomalies.

Proposition 2.2. There exists an isomorphism between $H^{1}\left(\mathscr{G}, \mathbb{C}^{*}(\mathscr{A})\right)$ and $H^{1}(\mathfrak{g}, \mathbb{C}(\mathscr{A}))$. 
Proof. Let $f(g ; A)$ be a representative of $[f] \in H^{1}\left(\mathscr{G}, \mathbb{C}^{*}(\mathscr{A})\right)$. Define

$$
a(\tau ; A)=: \lim _{t \rightarrow 0}\left[f\left(g_{t} ; A\right)-1\right] / t=d_{\tau} f,
$$

where $g_{t}=\exp (t \tau)$, for $\tau \in \mathfrak{g}$. Notice that $d_{\tau} f$ is the partial differential of $f$ with respect to $\mathscr{G}$ evaluated at the identity and computed along $\tau$. So it is linear in $\tau$ and depends smoothly on $A$, because $f$ is smooth on $\mathscr{G} \times \mathscr{A}$. Then $a(\tau ; A)$ is a 1 -cochain on $\mathfrak{g}$ with values in $\mathbb{C}(\mathscr{A})$. We now show that $\Delta f=1$ implies $\delta a=0$. CampbellHausdorff formulas tell us that

$$
g^{1}{ }_{t} g_{t}^{2}=\exp \left(t \tau_{1}\right) \exp \left(t \tau_{2}\right)=1+t\left(\tau_{1}+\tau_{2}+\frac{1}{2}\left[\tau_{1}, \tau_{2}\right]\right)+O\left(t^{2}\right) .
$$

Then

$$
\begin{aligned}
f\left(g^{1}{ }_{t} g_{t}^{2} ; A\right) & =1+t\left(a\left(\tau_{1}\right)+a\left(\tau_{2}\right)+\frac{1}{2} a\left(\left[\tau_{1}, \tau_{2}\right]\right)+O\left(t^{2}\right),\right. \\
f\left(g^{2}{ }_{t} ; A g^{1}{ }_{t}\right) & =1+t\left(a\left(\tau_{2}\right)+\mathscr{L}_{\tau_{1}} a\left(\tau_{2}\right)+O\left(t^{2}\right),\right. \\
f\left(g^{1}{ }_{t} ; A\right. & =1+t\left(a\left(\tau_{1}\right)+O\left(t^{2}\right) .\right.
\end{aligned}
$$

So, at first order in $t, \Delta f=1$ implies

$$
\mathscr{L}_{\tau_{1}} a\left(\tau_{2}\right)=\frac{1}{2} a\left(\left[\tau_{1}, \tau_{2}\right]\right) .
$$

Subtracting from this equation the same one with $\tau_{1}$ and $\tau_{2}$ exchanged, we get $\delta a=0$. This correspondence clearly induces a correspondence $[f] \leadsto[a]$ in cohomology, which is injective and a group homomorphism, because $d_{\tau}\left(f_{1} f_{2}\right)$ $=d_{\tau} f_{1}+d_{\tau} f_{2}$. We finally prove surjectivity, by showing that any $a(\tau: A)$ can be written as $d_{\tau} f$ for some 1 -cocycle $f$. For any $A \in \mathscr{A}$, let $i_{A}: \mathscr{G} \rightarrow \mathscr{A}$ be the injection of $\mathscr{G}$ onto the orbit through $A$ and consider the one form on $\mathscr{G}$ given by $i_{A}^{*} a$. Now, $d i{ }_{A}^{*} a$ $=i_{A}^{*} \delta a=0$, so $i_{A}^{*} a$ is closed on $\mathscr{G}$, and it is locally exact. We define a function

$$
F_{\gamma}(g ; A)=\int_{\gamma} i_{A}^{*} a,
$$

where $\gamma$ is a curve on $\mathscr{G}$ from $\mathbb{1}$ to $g$. Clearly, $F_{\gamma}$ depends on $\gamma$. Since the integrand is closed, $F_{\gamma^{\prime}}(g ; A)=F_{\gamma}(g ; A)$ if and only if the loop $\gamma^{\prime}-\gamma$ is homotopic to zero on $\mathscr{G}$. If not, $F_{\gamma^{\prime}}(A)=F_{\gamma}(g ; A)+n$, where $n$ can be normalized to be some integer depending only on the homotopy class of $\gamma^{\prime}-\gamma$. Then $\exp (2 \pi i F(g ; A))=f(g ; A)$ is independent of the chosen path and never vanishing on $\mathscr{G} \times \mathscr{A}$. Now, one can easily prove that $F_{\gamma}\left(g_{1} g_{2} ; A\right)=F_{\gamma_{1}}\left(g_{1} ; A\right)+F_{\gamma_{2}}\left(g_{2} ; A g_{1}\right)$, by simply splitting the integral into two pieces. Then $\Delta f=1$, and the correspondence $d: H_{1}\left(\mathscr{G}, \mathbb{C}^{*}(\mathscr{A})\right)$ $\rightarrow H^{1}(\mathscr{G}, \mathbb{C}(\mathscr{A}))$ is a surjective monomorphism and hence an isomorphism. Notice that the function $F(g ; A)$ is actually the so-called Wess-Zumino term [13].

\section{Topological Origin of Non-Abelian Anomalies}

Having introduced one more cohomology group $H^{1}\left(\mathscr{G}, \mathbb{C}^{*}(\mathscr{A})\right)$ to describe anomalies, our next task is to compute it. This seems to be impossible to achieve directly, nor is the Lie algebra cohomology more treatable. A standard way of computing cohomology groups is to show that they are isomorphic to some easier ones. This is the way we follow here by showing that $H^{1}\left(\mathscr{G}, \mathbb{C}^{*}(\mathscr{A})\right)$ is isomorphic to 
the Chech cohomology group $H^{2}(\mathcal{O}, Z)$ of the orbit space $\mathcal{O}$. We refer to Bott and $\mathrm{Tu}$ [16] for an introduction to Chech cohomology. As we shall see, this identification will give us a further isomorphism of $H^{1}\left(\mathscr{G}, \mathbb{C}^{*}(\mathscr{A})\right)$ with $\pi_{1}(\mathscr{G})$. Now, on $S^{4}$ one knows that $\pi_{1}(\mathscr{G})=\pi_{5}(G)$ [17], and the problem of classifying anomalies reduces to the study of the fifth homotopy group of a finite dimensional Lie group. This is explicitly known for the groups $G$ which enter in physical applications of gauge theories.

Our first step is to translate a one cocycle $f$ into some geometrical structure.

Proposition 3.1. A one cocycle $f$ representing a class $[f] \in H^{1}\left(\mathscr{G}, \mathbb{C}^{*}(\mathscr{A})\right)$ defines a unique line bundle L over the orbit space. If $f^{\prime}$ is another representative of $[f]$, the corresponding line bundle $L^{\prime}$ is isomorphic to $L$.

Proof. First, we construct $L$. Pick up a family of local sections $s_{\alpha}: U_{\alpha} \rightarrow \mathscr{A}$, trivializing the principal orbit bundle $\pi: \mathscr{A} \rightarrow \mathcal{O}$. Here $\left\{U_{\alpha}\right\}$ is a covering for $\mathcal{O}$. Any $A \in \pi^{-1}\left(U_{\alpha}\right)$ can be then represented by $=s_{\alpha}(\underline{A}) g(A)$, where $\underline{A}$ synthetically denotes the orbit $\pi(A)$. On $U_{\alpha} \cap U_{\beta}$, we have $s_{\beta}(\underline{A})=s_{\alpha}(\underline{A}) g_{\alpha \beta}(\underline{A})$, where $g_{\alpha \beta}: U_{\alpha} \cap U_{\beta} \rightarrow \mathscr{G}$ are transition functions for the orbit bundle. Given a representative $f$ of $[f] \in H^{1}\left(\mathscr{G}, \mathbb{C}^{*}(\mathscr{A})\right)$, the map $f_{\alpha \beta}: U_{\alpha} \cap U_{\beta} \rightarrow C^{*}$ given by

$$
f_{\alpha \beta}(\underline{A})=: f\left(g_{\alpha \beta}(\underline{A}) ; s_{\alpha}(\underline{A})\right)
$$

defines a cohomology class in $H^{1}\left(\mathcal{O}, \mathscr{C}^{*}\right)$, where $\mathscr{C}^{*}$ denotes the sheaf of germs of never vanishing complex valued functions on $\mathcal{O}$. To see that this is the case, it suffices to show that $f_{\alpha \beta}$ is a one Chech cocycle on $\mathcal{O}$, i.e. $f_{\alpha \beta}=f_{\alpha \beta}{ }^{-1}$ and $f_{\alpha \beta} f_{\beta \gamma} f_{\gamma \alpha}=1$. Indeed,

and

$$
\begin{aligned}
f_{\alpha \beta} & =f\left(g_{\alpha \beta} ; s_{\alpha}\right)=f\left(g_{\alpha \beta} ; s_{\beta} g_{\alpha \beta}{ }^{-1}\right) \\
& =f\left(1, s_{\beta}\right) / f\left(g_{\alpha \beta}{ }^{-1} ; s_{\beta}\right)=1 / f\left(g_{\beta \alpha}, s_{\beta}\right)=f_{\beta \alpha}{ }^{-1} .
\end{aligned}
$$

$$
\begin{aligned}
f_{\alpha \beta} f_{\beta \gamma} f_{\gamma \alpha} & =f\left(g_{\alpha \beta} ; s_{\alpha}\right) f\left(g_{\beta \gamma} ; s_{\beta}\right) f\left(g_{\gamma \alpha} ; s_{\gamma}\right) \\
& =f\left(g_{\alpha \beta} ; s_{\alpha}\right) f\left(g_{\beta \gamma} ; s_{\alpha} g_{\alpha \beta}\right) f\left(g_{\gamma \alpha} ; s_{\alpha} g_{\alpha \gamma}\right) \\
& =f\left(g_{\alpha \beta} ; s_{\alpha}\right)\left[f\left(g_{\alpha \beta} g_{\beta \gamma} ; s_{\alpha}\right) / f\left(g_{\alpha \beta} ; s_{\alpha}\right)\right]\left[f\left(g_{\alpha \gamma} g_{\gamma \alpha} ; s_{\alpha}\right) / f\left(g_{\alpha \gamma} ; s_{\alpha}\right)\right] \\
& =f\left(g_{\gamma \alpha}^{-1} ; s_{\alpha}\right) / f\left(g_{\alpha \gamma} ; s_{\alpha}\right)=1,
\end{aligned}
$$

where we used the fact that the transition functions $\left\{g_{\alpha \beta}\right\}$ satisfy the following properties: $g_{\alpha \beta}=g_{\beta \alpha}^{-1}$ and $g_{\alpha \beta} g_{\beta \gamma} g_{\gamma \alpha}=1$. Then the cocycle $f$ can be used as a set of transition functions to construct a line bundle $L$ over the orbit space $\mathcal{O}$ [18]. Next we show that the correspondence $f \rightarrow L$ does not depend on the trivialization $\left\{s_{\alpha}\right\}$. If $s_{\alpha}^{\prime}=s_{\alpha} g_{\alpha}$ is a new trivializing family of sections, then $g_{\alpha \beta}^{\prime}=g_{\alpha}^{-1} g_{\alpha \beta} g_{\beta}$ and hence:

$$
\begin{aligned}
f_{\alpha \beta}^{\prime} & =f\left(g_{\alpha \beta}^{\prime} ; s_{\alpha}^{\prime}\right)=f\left(g_{\alpha}^{-1} g_{\alpha \beta} g_{\beta} ; s_{\alpha} g_{\alpha}\right) \\
& =f\left(g_{\alpha \beta} g_{\beta} ; s_{\alpha}\right) f\left(g_{\alpha}^{-1} ; s_{\alpha} g_{\alpha}\right) \\
& =f\left(g_{\beta} ; s_{\alpha} g_{\alpha \beta}\right) f\left(g_{\alpha \beta} ; s_{\alpha}\right) f\left(g_{\alpha}^{-1} ; s_{\alpha} g_{\alpha}\right) \\
& =f\left(g_{\beta} ; s_{\beta}\right) f_{\alpha \beta} f^{-1}\left(g_{\alpha} ; s_{\alpha}\right) .
\end{aligned}
$$

So, $f_{\alpha \beta}^{\prime}$ is cohomologous to $f_{\alpha \beta}$ in $H^{1}\left(\mathcal{O}, \mathscr{C}^{*}\right)$ and the line bundle $L^{\prime}$ is isomorphic to $L$. A similar computation shows that any other representative $f^{\prime}$ of $[f]$ yields a line bundle $L^{\prime}$ isomorphic to $L$. Conversely, given a line bundle $L$ on $\mathcal{O}$ associated to the 
principal orbit bundle $\pi: \mathscr{A} \rightarrow \mathcal{O}$, the pull back bundle $\pi^{*} L$ is trivial on $\mathscr{A}$ and $L$ is given by the quotient $L=\mathscr{A} \times{ }_{r} \mathbb{C}$. Here $r: \mathscr{G} \rightarrow \mathbb{C}^{*}$ is some homomorphism which depends only on the isomorphism class of $L$. Now $r$ can be seen as a map of $\mathscr{G}$ into never vanishing constant functionals on $\mathscr{A}$ and, since $r\left(g_{1} g_{2}\right)=r\left(g_{1}\right) r\left(g_{2}\right)$, it defines a class in $H^{1}\left(\mathscr{G}, \mathbb{C}^{*}(\mathscr{A})\right)$.

As a first consequence of Proposition 3.1, one can show the following:

Proposition 3.2. For any $[f] \in H^{1}\left(\mathscr{G}, \mathbb{C}^{*}(\mathscr{A})\right)$ there exist functionals $W: \mathscr{A} \rightarrow \mathbb{C}$ such that $f(g ; A)=W(A g) / W(A)$.

Proof. Let $w: \mathcal{O} \rightarrow L$ be a global section of the line bundle $L$ corresponding to $f$ via Proposition 3.1, and denote by $w_{\alpha}: U_{\alpha} \rightarrow L$ its local representation over a covering $\left\{U_{\alpha}\right\}$ of $\mathcal{O}$. For any trivializing family $s_{\alpha}: U_{\alpha} \rightarrow \mathscr{A}$ define on $\pi^{-1}\left(U_{\alpha}\right)$ the complex valued function $W_{\alpha}$ by

$$
W_{\alpha}(A)=f\left(g(A) ; s_{\alpha}(\underline{A})\right) w_{\alpha}(\underline{A}),
$$

where $A=s_{\alpha}(\underline{A}) g(A)$ as above. This proves locally the proposition, in fact, one has:

$$
\begin{aligned}
W_{\alpha}\left(A g^{\prime}\right) & =W_{\alpha}\left(s_{\alpha}(\underline{A}) g(A) g^{\prime}\right)=f\left(g(A) g^{\prime} ; s_{\alpha}(\underline{A})\right) w_{\alpha}(\underline{A}) \\
& =f\left(g^{\prime} ; s_{\alpha}(\underline{A}) g(A)\right) f\left(g(A) ; s_{\alpha}(\underline{A})\right) w_{\alpha}(\underline{A}) \\
& =f\left(g^{\prime}, A\right) W_{\alpha}(A),
\end{aligned}
$$

because $f$ is a one-cocycle in $H^{*}\left(\mathscr{G}, \mathbb{C}^{*}(\mathscr{A})\right)$. Since $w_{\beta}(\underline{A})=f\left(g_{\alpha \beta}(\underline{A}) ; s_{\alpha}(\underline{A})\right) w_{\alpha}(\underline{A})$, the family $W_{\alpha}$ glues to yield a globally defined functional $W$ on $\mathscr{A}$. Clearly, $W$ is never vanishing, and $[f]$ is trivial, if and only if the $w_{\alpha}$ 's can be chosen to be never vanishing on $\mathcal{O}$. This cannot be the case, unless $L$ is trivial.

Remark. This proposition completes Proposition 2.1, yielding the result that the construction of one cocycles $f$ on $\mathscr{G}$ by means of suitable functionals $W$ on $\mathscr{A}$ exhaust the first cohomology group $H^{1}\left(\mathscr{G}, \mathbb{C}^{*}(\mathscr{A})\right)$.

Having constructed a line bundle $L \in H^{1}\left(\mathcal{O}, \mathscr{C}^{*}\right)$ corresponding to any $[f]$ in $H^{1}\left(\mathscr{G}, \mathbb{C}^{*}(\mathscr{A})\right)$, we can extract out of $L$ some information which is directly relevant for anomalies. Recall from the proof of Proposition 3.1 that $L$ is given as the quotient $L=\mathscr{A} \times{ }_{r} \mathbb{C}$, where $r: \mathscr{G} \rightarrow \mathbb{C}^{*}$ is an unique homomorphism. Clearly, for any $g \in \mathscr{G}, r(g)$ can be considered as a constant functional in $\mathbb{C}^{*}(\mathscr{A})$ and for any $\tau \in \mathfrak{g}$, the derived homomorphism $r_{*}: \mathfrak{g} \rightarrow \mathbb{C}$ defines a constant functional $r_{*}(\tau)$ in $\mathbb{C}(\mathscr{A})$. Then we have

Proposition 3.3. Let $r: \mathscr{G} \rightarrow \mathbb{C}^{*}$ be the homomorphism corresponding to $[f]$ in $H^{1}\left(\mathscr{G}, \mathbb{C}^{*}(\mathscr{A})\right)$. Then

i) $f$ and $r$ are cohomologous in $H^{1}\left(\mathscr{G}, \mathbb{C}^{*}(\mathscr{A})\right)$, i.e. there exists a never vanishing functional $N$ on $\mathscr{A}$ such that $f(g ; A)=r(g) N(A g) / N(A)$,

ii) the anomaly $a(\tau ; A)=d_{\tau} f$ is cohomologous to $r_{*}$ in $H^{1}(\mathfrak{g}, \mathbb{C}(\mathscr{A}))$.

Proof. We represent $f$ by $f(g ; A)=W(A g) / W(A)$ as in Proposition 3.2. Recall that this is done by extending in some suitable way a section $w: \mathcal{O} \rightarrow L$ of a line bundle $L=\mathscr{A} \times{ }_{r} \mathbb{C}$. Then pulling back $w$ on $\mathscr{A}$, we have that $\pi^{*} w(A g)=r(g) \pi^{*} w(A)$. Recall that the functional $W(A)$ gotten by extending $w$ as in Proposition 3.2 is given by $W(A)=N(A) \pi^{*} w(A)$, where $N(A)$ is never vanishing. Accordingly,

$$
f(g ; A)=N(A g) \pi^{*} w(A g) /\left(N(A) \pi^{*} w(A)\right)=r(g)(\Delta N)(g ; A),
$$


and

$$
a(\tau ; A)=\mathrm{d}_{\tau} f=r_{*}(\tau)+(\delta \log N)(\tau),
$$

where $\log N$ exists because $N$ is never vanishing on $\mathscr{A}$.

The one-to-one correspondence between non-abelian anomalies and line bundles over the orbit space given by the propositions above is the basic result which allows an explicit computation of the group $H^{1}\left(\mathscr{G}, \mathbb{C}^{*}(\mathscr{A})\right)$ or, which is the same, $H^{1}(\mathfrak{g}, \mathbb{C}(\mathscr{A}))$. Indeed, as we have seen, both of them are isomorphic to the group $H^{1}\left(\mathcal{O}, \mathscr{C}^{*}\right)$ of line bundles over $\mathcal{O}$.

To compute this last group, we recall [18] that it is isomorphic to the Chech cohomology group $H^{2}(\mathcal{O}, Z)$. In fact, from the exponential sequence of sheaves

$$
0 \longrightarrow Z \longrightarrow \mathscr{C} \stackrel{\exp 2 \pi i}{\longrightarrow} \mathscr{C}^{*} \longrightarrow 1
$$

(where $Z, \mathscr{C}, \mathscr{C}^{*}$ denote the sheaves of integer, complex and never vanishing complex valued functions on $\mathcal{O}$ ), one has that

$$
0=H^{1}(\mathcal{O}, \mathscr{C}) \rightarrow H^{1}\left(\mathcal{O}, \mathscr{C}^{*}\right) \rightarrow H^{2}(\mathcal{O}, Z) \rightarrow H^{2}(\mathcal{O}, \mathscr{C})=0,
$$

where $H^{k}(\mathcal{O}, \mathscr{C})=0$ for $k \geqq 1$, because $\mathscr{C}$ is a fine sheaf.

Now, $H^{2}(\mathcal{O}, Z)$ is in turn isomorphic to $H^{1}(\mathscr{G}, Z)$. To see why this is the case, we notice that from the long homotopy sequence of the orbit bundle $\mathscr{G} \rightarrow \mathscr{A} \rightarrow \mathcal{O}$ one has that $\pi_{2}(\mathcal{O})=\pi_{1}(\mathscr{G})$ and $\pi_{1}(\mathcal{O})=\pi_{0}(\mathscr{G})$. Now, if $\mathscr{G}$ is connected, it is also path connected and by Hurewicz theorem (see e.g. [16, p. 125]) $H^{1}(\mathscr{G}, Z)=\pi_{1}(\mathscr{G})$. Also, the orbit space $\mathcal{O}$ is connected and simply connected. Hence, again from Hurewicz theorem, $H^{1}(\mathcal{O}, Z)=0, H^{2}(\mathcal{O}, Z)=\pi_{2}(\mathcal{O})=\pi_{1}(\mathscr{G})=H^{1}(\mathscr{G}, Z)$.

Summing up, we collect the results above in the following:

Theorem 3.4. There exist isomorphisms

$$
H^{1}(\mathfrak{g}, \mathbb{C}(\mathscr{A}))=H^{1}\left(\mathscr{G}, \mathbb{C}^{*}(\mathscr{A})\right)=H^{1}\left(\mathcal{O}, \mathscr{C}^{*}\right)=H^{2}(\mathcal{O}, Z)=H^{1}(\mathscr{G}, Z) .
$$

So far we have been jumping from one cohomology group to another. Now we show that there is some merit in such a procedure, because $H^{1}(\mathscr{G}, Z)$ can be in principle computed. In particular, the following result holds:

Proposition 3.5. Let $\mathscr{G}$ be a Hilbert Lie group of pointed G-valued gauge transformations on $S^{4}$, where $G$ is a compact Lie group such that $\pi_{4}(G)=0$. Then $H^{1}(\mathscr{G}, Z)=\pi_{5}(G)$.

Proof. From Hurewicz theorem we know that $H^{1}(\mathscr{G}, Z)=$ abelianization of $\pi_{1}(\mathscr{G})$. Now on $S^{4}, \mathscr{G}$ has the homotopy type of $\operatorname{Map}_{0}\left(S^{4}, G\right)[19]$; accordingly, $\pi_{i}(\mathscr{G})$ $=\pi_{i+4}(G)$. Then $\pi_{1}(\mathscr{G})$ is abelian and the proposition is proved.

Remark. For instance, if $G=\mathrm{SU}(n)$ or $\mathrm{U}(n)$ for $n \geqq 3$, then $H^{1}(\mathscr{G}, Z)=Z$, because $\pi_{5}(\mathrm{SU}(n))=\pi_{5}(\mathrm{U}(n))=Z$. In this case we see that the "topological" part of the anomaly is unique up to a multiplicative constant. Indeed, if $r$ is the homomorphism of $\mathscr{G}$ in $\mathrm{U}(1)$ corresponding to the generator of $H^{1}(\mathscr{G}, Z)$, then any other homomorphism $r^{\prime}$ is given by $r^{\prime}=r^{m}$, for some $m \in Z$. Then $r^{\prime}{ }_{*}=m r_{*}$ and the anomalies are proportional, i.e. $a^{\prime}(\tau ; A)=m a(\tau ; A)$.

Finally, we can make contact with secondary invariants by the usual transgression process [5], which is implicit in Theorem 3.4. We have seen that the 
"topological" part of the anomaly corresponds to a line bundle $L$ over the orbit space $\mathcal{O}$. This line bundle can be described either as an element in $H^{1}\left(\mathcal{O}, \mathscr{C}^{*}\right)$ or as a class in $H^{2}(\mathcal{O}, Z)$. The latter is called the first Chern class of $L$ [18]. On "good" manifolds, as all the manifolds considered here, Chech cohomology and De Rham cohomology are isomorphic. Then the first Chern class of $L$ can be represented by a closed differential 2-form $c_{1}$ on $\mathcal{O}$. Pulling back $c_{1}$ from $\mathcal{O}$ to $\mathscr{A}$, one gets an exact form, i.e. $\pi^{*} c_{1}=d T c_{1}$, where $d$ now denotes the differential on $\mathscr{A}$. The 1 -form $T c_{1}$ on $\mathscr{A}$ is called the transgression of $c_{1}$. It is also known that when restricted to an orbit of $\mathscr{G}$ in $\mathscr{A}, T c_{1}$ defines a cohomology class in $H^{1}(\mathscr{G}, Z)$; so we get the isomorphism $H^{1}(\mathscr{G}, Z)=H^{2}(\mathcal{O}, Z)$ in terms of differential forms. This tells us that

Proposition 3.6. The "topological" part of the anomaly is given by vertically restricting to an orbit of $\mathscr{G}$ in $\mathscr{A}$ the transgression of the first Chern class $c_{1}$ of $L$.

Accordingly one can identify the anomaly with some differential form. The subtle problem is the actual construction of this form. This can be done by a closer inspection of the vacuum functional $W[9,10]$; for instance, for chiral fermions, one gets that $L$ is the determinant line bundle of the index bundle for the Dirac operator. Then a direct computation of the first Chern class $c_{1}$ of $L$ and of its transgression $T c_{1}$ can be achieved via the topological part of the family index theorem of Atiyah and Singer [7]. As it is known, this yields exactly the same form for non-abelian anomalies as given by perturbative techniques [7].

\section{Conclusions}

To summarize, we recall all the equivalent ways of looking at non-abelian anomalies considered in this paper:

i) A non-trivial cohomology class $a=a(\tau ; A)$ in $H^{1}(\mathfrak{g}, \mathbb{C}(\mathscr{A}))$ given by $a(\tau ; A)$ $=\left(\mathscr{L}_{\tau} W\right) / W$, which expresses that the generating functional $W$ is not $\mathscr{G}$-invariant and vanishes along some orbit.

ii) A non-trivial cohomology class $f(g ; A)=W(g A) / W(A)$ in $H^{1}\left(\mathscr{G}, \mathbb{C}^{*}(\mathscr{A})\right)$, again reflecting that $W$ vanishes somewhere.

iii) A line bundle $L$ on the orbit space $\mathcal{O}$, such that $W(A)=N(A) \pi^{*} w(A)$, where $w: \mathcal{O} \rightarrow L$ is some section of $L$.

iv) A non-trivial cohomology class in $H^{1}(\mathscr{G}, Z)$, corresponding to $L$ via transgression.

While the first two descriptions are related to abstract Lie algebra or group cohomologies, the last two involve only topology.

The main result of this paper was to show how non-abelian anomalies can be directly related to the topology of the group of gauge transformations. This has been known for some time, see e.g. [20], but a direct link between the homotopy group $\pi_{1}(\mathscr{G})$ and unicity of non-abelian anomalies was missing. By avoiding restrictions to local functionals, we computed the relevant cohomology group, which turns out to be isomorphic to $\pi_{1}(\mathscr{G})$. If it is $Z$, as in gauge theories over $S^{4}$ with gauge group $\mathrm{SU}(n), n \geqq 3$, all these non-abelian anomalies are integer multiples of a basic one. Clearly, this integer coefficient cannot be fixed by abstract arguments, but explicit computations are needed. In any case, whenever $\mathscr{G}$ is 
connected and $\pi_{1}(\mathscr{G})=Z$, the non-abelian anomaly is "essentially" unique. This fully matches with the index theorem approach [7].

Also we computed a part of the first BRS cohomology group, namely the part due to the topology of the group of gauge transformations. Notice that the exponential version of this cohomology considered here easily extends to the case of gravitational anomalies, when Lie algebra cohomology is a hard job, because the group of diffeomorphisms is only a Frechét-Lie group.

As these "topological" anomalies cannot be eliminated by using the whole space of smooth functionals over $\mathscr{A}$, no doubt that they still sit there when one restricts to the physical case of functionals locally depending on gauge potentials. Clearly, one gets more anomalies with such a restriction, but none of the nonabelian type. In particular, the occurrence of abelian anomalies in quantum field theory draws us back to study local BRS cohomology. However, one may notice that:

a) Abelian anomalies actually arise in terms of global group actions; for instance one does not need to gauge chiral U(1) symmetry or dilatations.

b) For power-counting renormalizable theories, the space of local functionals should be restricted to a finite dimensional linear space generated by the functionals having the correct physical dimensions of an action.

Thus the study of the unicity problem for abelian anomalies in BRS cohomology can be tackled via explicit computations. As it is well known, some of these anomalies are due to the topology of finite dimensional manifolds, but it seems that, at least for the case of trace anomalies, topology alone does not control the whole anomaly structure, as shown for instance in [21].

Finally, we would like to add some physical comments. Consider a path integral of the form

$$
\mathscr{I}=\int \mathscr{D} A(\ldots) e^{-S_{\mathbf{Y M}}(A)} \int \mathscr{D} \psi \mathscr{D} \bar{\psi} e^{-S(\bar{\psi}, \psi ; A)},
$$

which gives the expectation value of some physical observable (...) in a gauge theory with chiral fermions $\psi, \bar{\psi}$. The fermion path integral can be explicitly computed yielding after regularization the vacuum functional $W=W(A)$ considered above. Thus one has

$$
\mathscr{I}=\int \mathscr{D} A(\ldots) e^{-S_{\mathrm{YM}}(A)} W(A),
$$

and one would like to write $W(A)$ as the exponential of an effective action $\Gamma=\Gamma(A)$ to get

$$
\mathscr{I}=\int \mathscr{D} A(\ldots) e^{-\left(S_{\mathbf{Y M}}(A)+\Gamma(A)\right)} .
$$

If $\Gamma(A)$ were $\mathscr{G}$-invariant, one could consistently factor out the integration on gauge equivalent potentials by inserting a gauge fixing term and the relative Faddev-Popov determinant [22]. Then one can prove renormalizability and unitarity as usual.

The occurrence of anomalies can break this procedure at two levels. First of all, as we have seen, non-abelian anomalies imply that the effective action $\Gamma(A)$ is not well defined, so one should work with the form (21) of the path integral. Clearly, 
this makes it impossible to define a perturbative series. Accordingly there is no surprise that these anomalies can be seen perturbatively, although their origin is by no means of perturbative nature. Notice also that, since $W(A)$ is not $\mathscr{G}$-invariant, there is little motivation to introduce a gauge fixing and Faddev-Popov ghosts.

Secondly, when $\Gamma(A)$ exists, that is non-abelian anomalies are absent, one can apply perturbative techniques to study the path integral in the form (22). However, $\Gamma(A)$ may not be $\mathscr{G}$-invariant, and one seeks for a local counterterm $\Lambda_{\text {loc }}(A)$ to be added to the Yang-Mills action in order to restore the gauge invariance of the total action $S_{\mathrm{YM}}+\Lambda_{\text {loc }}+\Gamma$. Here "local" anomalies of the abelian type arise and local BRS cohomology is effective. Notice that gauge invariance is turned into BRS invariance after the Faddev-Popov trick is performed.

From these remarks it is clear that non-abelian anomalies forbid the perturbative study of quantum field theories. It is tempting to wonder whether some future non-perturbative approach might give some physical meaning to these topological effects, as it is at present done for abelian anomalies via perturbative techniques.

Acknowledgements. We thank R. Annunziata, R. Catenacci, P. Cotta-Ramusino, M. Martinelli, P. Pirola, and R. Stora for suggestions and discussions.

\section{References}

1. See e.g. Adler, S.: In: Lectures on elementary particles and quantum field theory, Deser, S. et al. (eds.). MIT Press (1970) and Jackiw, R.: In: Lectures on current algebra and its applications. Princeton, NJ: Princeton University Press 1972, and references quoted therein

2. See e.g. Stora, R.: Continuum gauge theories. In: New developments in quantum field theories and statistical mechanics. Levy, H., Mitter, P. (eds.). New York: Plenum Press 1977, and references quoted therein

3. For the mathematical set up of this cohomology see Bonora, L., Cotta-Ramusino, P.: Some remarks on BRS transformations, anomalies, and the cohomology of the Lie algebra of the group of gauge transformations. Commun. Math. Phys. 87, 589 (1983)

4. See e.g. Becchi, C., Rouet, A., Stora, R.: In: Renormalization theory, Velo, G., Wightman, A.S. (eds.). Amsterdam: Reidel 1976, and references quoted therein

5. Chern, S.S., Simons, J.: Characteristic forms and geometric invariants. Ann. Math. 99, 48 (1974)

6. For an introduction, see e.g. Jackiw, R., Rebbi, C.: Spinor analysis of Yang-Mills theory. Phys. Rev. D 16, 1052 (1977)

7. Atiyah, M.F., Singer, I.M.: Dirac operators coupled to vector potentials. Proc. Natl. Acad. Sci. 81, 2597 (1984)

8. Alvarez, O., Singer, I.M., Zumino, B.: Gravitational anomalies and the family index theorem. Commun. Math. Phys. 96, 409 (1984)

9. Alvarez-Gaumé, L., Ginsparg, P.: The topological meaning of non-abelian anomalies. Nucl. Phys. B 243, 449 (1984)

10. Martellini, M., Reina, C.: Some remarks on the index theorem approach to anomalies. Milano: Ann. Inst. H. Poincaré (in press) (1985)

11. Palais, R.S.: Foundations of global non-linear analysis. New York: Benjamin 1968

12. Cotta-Ramusino, P., Reina, C.: The action of the group of bundle automorphisms on the space of connections and the geometry of gauge theories. J. Geom. Phys. 1, 121 (1984)

13. Faddeev, L.D., Raiman, A.G., Semenov-Tjan-Shansky, M.A.: Quantum anomalies and cocycles on the gauge group. Leningrad preprint (1984) 
14. Zumino, B.: Chiral anomalies and differential geometry. Les Houches Lectures (1983)

15. Wess, J., Zumino, B.: Consequences of anomalous Ward identities. Phys. Lett. 73 B, 95 (1971)

16. Bott, R., Tu, L.W.: Differential forms in algerbaic topology. GTM-82, Berlin, Heidelberg, New York: Springer 1982

17. Singer, I.M.: Some remarks on the Gribov ambiguity. Commun. Math. Phys. 60, 7 (1978)

18. See e.g. Morrow, J., Kodaira, K.: Complex manifolds. New York: Holt, Rinehart, and Winston 1971

19. Atiyah, M.F., Jones, J.D.S.: Topological aspects of Yang-Mills theory. Commun. Math. Phys. 61, 97 (1978)

20. Witten, E.: Global aspects of current algebra. Nucl. Phys. B 223, 422 (1983)

21. Bonora, L., Cotta-Ramusino, P., Reina, C.: Conformal anomaly and cohomology. Phys. Lett. 126 B, 305 (1983)

22. Abers, E.S., Lee, B.W.: Gauge theories. Phys. Rep. C9, 1 (1973)

23. Sumitani, T.: Derivation of chiral and gravitational anomalies from the generalized index theorem. University Tokyo preprint, Dec. 1984

Communicated by A. Jaffe

Received May 13, 1985 
